\title{
Correction to: Effects of COVID-19 Confinement on the Household Routines of Children in Portugal
}

\author{
André Pombo $\mathbb{D}^{1,2} \cdot$ Carlos Luz $^{2} \cdot$ Luis Paulo Rodrigues ${ }^{3,4} \cdot$ Rita Cordovil $^{1,5}$ \\ Published online: 31 December 2021 \\ (c) Springer Science+Business Media, LLC, part of Springer Nature 2022
}

Correction to: Journal of Child and Family Studies (2021) 30:1664-1674

https://doi.org/10.1007/s10826-021-01961-z

The reference \#7 of the manuscript is not correct. Where it's written "Chen, P., Mao, L., Nassis, G. P., Harmer, P., Ainsworth, B. E., \& Li, F. (2020). Wuhan coronavirus (2019-nCoV): The need to maintain regular physical activity while taking precautions. In Journal of Sport and
Health Science (Vol. 9, Issue 2, pp. 103 -104). Elsevier B. V. https://doi.org/10.1016/j.jshs.2020.02.001" should be: "Chen P, Mao L, Nassis GP, Harmer P, Ainsworth BE, Li F. Coronavirus disease (COVID-19): The need to maintain regular physical activity while taking precautions. J Sport Health Sci 2020;9(2):103-104. https://doi.org/10.1016/j. jshs.2020.02.001".

The original article has been corrected.
The original article can be found online at https://doi.org/10.1007/ s10826-021-01961-z.

André Pombo

apombo@eselx.ipl.pt

1 Faculdade de Motricidade Humana, Universidade de Lisboa, CruzQuebrada 1499-002, Portugal

2 Escola Superior de Educação, Instituto Politécnico de Lisboa, Lisboa 1549-003, Portugal

3 Escola Superior Desporto e Lazer de Melgaço, Instituto Politécnico de Viana do Castelo, Melgaço 4960-320, Portugal

4 Research Center in Sports Sciences Health Sciences and Human Development, CIDESD, Covilhã, Portugal

5 CIPER, Faculdade de Motricidade Humana, Universidade de Lisboa, Cruz-Quebrada 1499-002, Portugal 